\title{
Analytical solution of tank drainage for electrically conducting power law fluid
}

\author{
K. N. Memon ${ }^{1,3^{*}}$, A. M. Siddiqui ${ }^{2}$, Syed Feroz Shah ${ }^{1}$, S. Islam ${ }^{4}$ \\ ${ }^{1}$ Department of Basic Science, Mehran University of engineering technology jamshoro, Pakistan \\ ${ }^{2}$ Pennsylvania State University, York Campus, Edgecombe 17403, USA \\ ${ }^{3}$ Department of Mathematics and Statistics, QUEST, Nawabshah, Pakistan \\ ${ }^{4}$ Mathematics, Abdul Wali Khan University Mardan, KP Pakistan.
}

saeedislam@awkum.edu.pk

\begin{abstract}
This paper investigates the tank drainage problem of an isothermal, unsteady, incompressible electrically conducting Power law fluid. Analytic solution have been obtained from governing continuity and momentum equations subject to appropriate boundary conditions by using Perturbation method. The Power law fluid model solution without MHD is retrieved from this proposed model on substitution $\varepsilon=0$. Declaration on behalf of velocity profile, volume flux, average velocity, connection of time with respect to length of the tank and requirement of time for whole drainage of fluid are acquired. Special effects of numerous emerging parameter's on velocity profile $v_{z}$ and depth $H(t)$ of the fluid in the tank are graphically presented.
\end{abstract}

Keywords: Tank drainage, Power law MHD fluid, Analytical solution.

\section{INTRODUCTION}

In current years, non-Newtonian fluids have increase significant consideration on account of their numerous biological, industrial and technological applications. Here few cases of nonNewtonian fluids such as tooth paste, drilling mud, greases, paints, blood, polymer melts, clay coatings etc. It is an expansive class of fluids so; there is no any single model that can handle all the properties of such fluids as is done by the Newtonian fluids (described by the well-known Navier-Stokes equation). In this regard, several constitutive equations have been proposed to predict the physical structure and behavior of such types of fluids for different materials [1-2]. 
Presently the class of non-Newtonian fluids, the power law model have been broadly concentrated on account of numerical effortlessness and far reaching modern applications. Amid the last four decades, critical advancement has been acknowledged in the improvement of diagnostic arrangement and numerical calculations of power law liquid stream issues [3-5].

The drainage of a fluid through pipe of a tank under the action of gravity is an old, however complicated problem. The tank may be drained by an attach pipe or may be drained through evenhanded hole "orifice situation". The pipe possibly could be horizental or vertical or may contain a complete piping system with horizental extension and vertical drop with fittings and valve, etc. Usually tank has a shape of cylinderical contain a vertical wall however bottom may be conical hemisherical or by flat or might be additional shape. There is sometimes intrest in draining the tank should be totally dry in which situation the bottom shape needs to be accounted for and occasionally not.

Classifications of gravity draining fluid's are used extensively throughout industries, a small number of such classifications are: draining condensate into storage, water distribution, waste water management and dams, retrieval of chemicals from tank farm. The generated model will accurately represent tank draining behavior for all tanks with a similar setup. End effects, accuracy of time measurement, accuracy of height measurements and friction losses will be taken into consideration [6].

To day science due to practical concentration, the study of tank drainage flow has received significant consideration. Numerous analysts have pondered the break down these tpes of flows since their formulation. The power fluid's model have been utilized for tank drainage flow by [11] to investigate and solve the problem exactly. For simple viscous fluid, the theory depicting the efflux time concerning a tank has been efflux time of a tank has been inferred by Crosby [7] and by Bird, Stewart, and Lightfoot [8], and additionally extended to systems with the installed fittings by Hanesian [9]. It is founder fact that, when fluid is drained by mean of hole from the tank, the equation of Torricelli's is utilized to define the discharge velocity field and flow rate that is given [10-11], these types of the issues further revisited in [12]. For the turbulent flow at the exit pipe, the relation amongst the height of the fluid to the bottom of the exit pipe and the efflux time is calculated by [13]. Further a short note on mechanics of the slow draining for large tank is written by [14]. Two dimensional and two layer for rectangular tank draining 
unsteady flows is given in [15] and three dimensional for two fluid in the circular tank is system by [16]. Efflux Time and comparison of cylindrical with differential is specified in [17-20] and slow draining under the action of gravity for large spherical tank is studied in [21], they have compare the mathematical and experimental values and establish to be in good arrangement with the model. Usage of polymer solution's for drag reduction under the action of gravity is particular in [22-23].

In this article, we considered the problem of tank drainage for Power law MHD fluid. Analytical solutions of the consequential differential equations focus to boundary conditions, are found by using perturbation method and the substitution perturbation parameter $\varepsilon=0$, we retrieve the results for Power law fluid without MHD [11]. Also relationships for velocity profile, flow rate, average-velocity, depth of fluid in the tank at any time and time requirement of time for to complete drainage are considered. As per the best of our insight, the solution of the problem has not been accounted for in the literature.

This paper is structured by means of follows: Section number 2 provides basic governing equation's for Power law MHD fluid. Section number 3 deals with formulation and the solution of problem. Section number 4 deals with "flow rate", "average velocity", relationships how does the length of fluid change's with respect to time and requirement of time for to complete drainage. Results and discussion are specified in section number 5, finally conclusions are delivered in section number 6 .

\section{Basic Equations}

Essential governing equations for incompressible Power law MHD fluid flow, disregarding thermal effects are:

$$
\begin{gathered}
\nabla \cdot \mathbf{V}=0, \\
\rho \frac{D \mathbf{V}}{D t}=-\nabla p+\rho \mathbf{b}+\nabla \cdot \mathbf{T}+(\mathbf{J} \times \mathbf{B}),
\end{gathered}
$$


The symbol $\rho$ represents constant density, $p$ stand for the dynamic pressure, $\mathbf{V}$ be the velocity vector, $\mathbf{b}$ represent to the body force, $\mathbf{T}$ stands for the extra stress tensor and the operator $\frac{D}{D t}$ denotes the material derivative. As a result Lorentz force per unit volume be

$$
\mathbf{J} \times \mathbf{B}=\left[0,0,-\sigma B_{0}^{2} v_{z}\right],
$$

where $\sigma$ is the electrical conductivity, $\mathbf{B}=\left[0,0, B_{0}\right]$ be the uniform magnetic field, here $B_{0}$ be the applied magnetic field and $\mathbf{J}$ be the current density $\mathbf{J}$, which is

$$
\begin{gathered}
\mathbf{J}=\sigma[\mathbf{E}+\mathbf{V} \times \mathbf{B}], \\
\nabla \times \mathbf{B}=\mu_{0} \mathbf{J} .
\end{gathered}
$$

Here $\mathbf{E}$ is the electric field which is not considered in this study and $\mu_{0}$ be the magnetic permeability [24-27]. The extra stress tensor describing a Power law fluid [4-5] is made by:

$$
\mathbf{T}=\mu_{e f f} \mathbf{A}_{1}
$$

and

$$
\mu_{\text {eff }}=\eta\left|\sqrt{\frac{\mathbf{A}_{1}: \mathbf{A}_{1}}{2}}\right|^{n-1}
$$

here $\eta$ represent consistency coefficient, $n$ is the power-law index and $\mathbf{A}_{\mathbf{1}}$ be the $1^{\text {st }}$ Rivlin Ericksen tensor, represented as:

$$
\mathbf{A}_{1}=\nabla \mathbf{V}+(\nabla \mathbf{V})^{T}
$$

\section{Tank drainage}

Think about a tank of cylindrical shape having an incompressible Power law MHD fluid. Let suppose the radius of the tank is $R_{T}$, diameter of the tank be $D$ and $H_{0}$ be the initial depth of the fluid in the tank. The fluid which is present in the tank, which is drained down through by cylindrical pipe having length $L$ and radius be $R$. Promote all the more letting $H(t)$ be the depth of fluid in the tank at at all the time $t$.

Our strategy is to determine the velocity, pressure, volume flux, average velocity, relationship how does the time fluctuate with length and the time required for finish drainage. Here we take 
cylindrical coordinate's $(r, \theta, z)$ with $r$-axis normal to cylindrical pipe and $z$-axis along the center of the pipe in vertical direction. As the flow is only into the direction of $z$, the $r$ and $\theta$ component's of velocity field are equal to zero,

$$
V=\left[v_{r}, v_{\theta}, v_{z}\right]=\left[0,0, v_{z}(r, t)\right]
$$

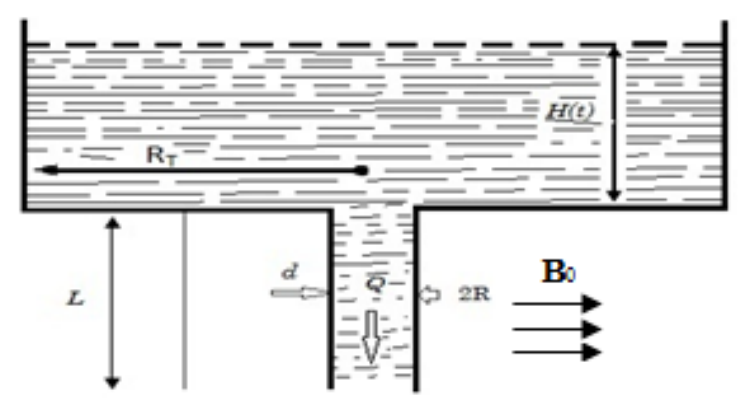

Figure 1: Geometry of the tank drainage flow down through pipe.

Utilizing velocity field (8), the equation of continuity (1) is indistinguishably fulfilled and the momentum equation (2) dimenshion toward

$$
\begin{array}{cl}
r \text {-component of momentum: } & \frac{\partial p}{\partial r}=0 \\
\theta \text {-component of momentum: } & \frac{1}{r} \frac{\partial p}{\partial \theta}=0 \\
z \text {-component of momentum: } \rho \frac{\partial v_{z}}{\partial t}=-\frac{\partial p}{\partial z}+\frac{\eta}{r} \frac{\partial}{\partial r}\left[r\left|\frac{\partial v_{z}}{\partial r}\right|^{n-1} \frac{\partial v_{z}}{\partial r}\right]+\rho g-\sigma B_{0}^{2} v_{z}(r) .
\end{array}
$$

According to definition of magnitude needs that the result be a positive number. Thus we sellect of sign that produces $\left|\frac{\partial v_{z}}{\partial r}\right|$ in equation (11) depends on whether the derivative $\frac{\partial v_{z}}{\partial r}$ is positive or negative. In the current example as $r$ increases, the velocity decreases - the velocity is at its maximum at the center of the pipe. The derivative $\frac{\partial v_{z}}{\partial r}$ is negative, and therefore $\left|\frac{\partial v_{z}}{\partial r}\right|=-\frac{\partial v_{z}}{\partial r}$. 
From equations ( 9 - 10) we can see that the equation of motion is now quite simple, yielding that the pressure is only function of $z$ and $t$ and the equation to be solved for $v_{z}(r, t)$ is

$$
\rho \frac{\partial v_{z}}{\partial t}=-\frac{\partial p}{\partial z}-\frac{\eta}{r} \frac{\partial}{\partial r}\left[r\left(-\frac{\partial v_{z}}{\partial r}\right)^{n}\right]+\rho g-\sigma B_{0}^{2} v_{z}(r)
$$

Equation (12) is a partial differential equation for $p$ and $v_{z}$. The velocity remains nearly constant with time in the pipe flow due to slow draining such that we may neglect time derivative $\frac{\partial v_{z}}{\partial t}$. Also flow be in the pipe is due to both hydrostatic pressure and gravity, at the pipe entrance and exit the pressure is,

$$
\begin{aligned}
& \text { at } \quad z=0, \quad p=p_{1}=\rho g H(t), \\
& \text { at } \quad z=L, \quad p=p_{2}=0,
\end{aligned}
$$

so that

$$
\frac{\partial p}{\partial z}=-\frac{\rho g H(t)}{L}
$$

The equation of motion (12) thus reduces to

$$
\frac{1}{r} \frac{\partial}{\partial r}\left[r\left(-\frac{\partial v_{z}}{\partial r}\right)^{n}\right]=\frac{\rho g}{\eta}\left[\frac{H(t)}{L}+1\right]-\frac{\sigma B_{0}^{2}}{\eta} v_{z}(r)
$$

The related boundary conditions are

$$
\begin{aligned}
& \text { at } \quad r=0, \quad \frac{\partial v_{z}}{d r}=0, \\
& \text { at } \quad r=R, \quad v_{z}=0
\end{aligned}
$$

\section{Perturbation solution:}

We take $\varepsilon=\frac{\sigma B_{0}^{2}}{\eta}$ to be a small parameter and velocity profile $v_{z}(r, \varepsilon)$ can be stated as a power series given by,

$$
v_{z}(r, \varepsilon) \approx v_{0}(r)+\varepsilon v_{1}(r)+\varepsilon^{2} v_{2}(r)+\ldots
$$

By utizing equation (17) into the equation (14), (15) and (16) and equating coefficients of like power's of $\varepsilon$, we acquire the following set of problems along with their associated boundary 
condition's:

\section{zeroth order problem:}

$$
\varepsilon^{0}: \frac{1}{r} \frac{\partial}{\partial r}\left[r\left(-\frac{\partial v_{0}}{\partial r}\right)^{n}\right]=\frac{\rho g}{\eta}\left[\frac{H(t)}{L}+1\right]
$$

with related boundary conditions,

$$
\begin{aligned}
\frac{d v_{0}}{d r}=0 & \text { at } & r=0, \\
v_{0}=0 & \text { at } & r=R .
\end{aligned}
$$

First order problem:

$$
\varepsilon^{1}: \frac{1}{r} \frac{d}{d r}\left(r n\left(-\frac{d v_{0}}{d r}\right)^{n-1}\left(-\frac{d v_{1}}{d r}\right)\right)-v_{0}==0,
$$

through belonging conditions,

$$
\begin{array}{lll}
\frac{d v_{1}}{d r}=0 \quad \text { at } & r=0, \\
v_{1}=0 \quad \text { at } & r=R .
\end{array}
$$

\section{Velocity profile:}

Zeroth order solution:

The solution of equation (18) by mean of boundary conditions from equations (19) and (20) is

$v_{0}=\frac{n}{n+1}\left[\frac{\rho g}{2 \eta L}(H(t)+L)\right]^{\frac{1}{n}}\left(R^{\frac{1}{n}+1}-r^{\frac{1}{n}+1}\right)$.

First-order solution:

Replacing the zeroth order solution from equation (24), into equation (21) and subject to conditions from equation (22) and (23) is specified by

$v_{1}=-\frac{n}{2(n+1)^{2}}\left[\frac{\rho g}{2 \eta L}(H(t)+L)\right]^{\frac{2}{-1}-1}\left[R^{\frac{1}{n}+1}\left(r^{\frac{1}{n}+1}-R^{\frac{1}{n+1}}\right)-\frac{n}{(1+3 n)}\left(r^{\frac{2}{n}+2}-R^{\frac{2}{n}+2}\right)\right]$

Thus the solution with perturbation technique correct upto first order is,

$V_{z}=\frac{n}{n+1}\left[\frac{\rho g}{2 \eta L}(H(t)+L)^{\frac{1}{n}}\left(R^{\frac{1}{n}+1}-r^{\frac{1}{n}+1}\right)-\frac{n}{2(n+1)^{2}}\left[\frac{\rho g}{2 \eta L}(H(t)+L)^{\frac{2}{n}-1}\left[R^{2^{\frac{1}{n}+1}}\left(r^{\frac{1}{n}+1}-R^{\frac{1}{n}+1}\right)-\frac{n}{(1+3 n)}\left(r^{\frac{2}{n}+2}-R^{\frac{2}{n}+2}\right)\right]\right.\right.$

Here important note that if we select to the perturbation parameter $\varepsilon=0$ in equation (26), we get 
the solution for same problem with Power law fluid without MHD [11] and for setting $\varepsilon=0$ and $n=1$, we get the solution for the Newtonian withought MHD fluid [28].

\section{Flow rate, average velocity and time required for to complete drainage}

The "flow rate $Q$ " per unit width is specified through the formula

$$
Q=\int_{0}^{R} 2 \pi r v_{z}(r, t) d r
$$

Using velocity profile (26) in equation (27), one can calculate the flow rate

$$
Q=\frac{n \pi}{1+3 n}\left[\left(\frac{\rho g}{2 \eta L}(H(t)+L)\right)^{\frac{1}{n}} R^{\frac{1}{n}+3}+\frac{\varepsilon}{2(1+2 n)}\left(\frac{\rho g}{2 \eta L}(H(t)+L)\right)^{\frac{2}{n}-1} R^{\frac{2}{n}+4}\right] .
$$

We determine the average velocity, $\bar{V}$ by utilising the formula

$$
\bar{V}=\frac{Q}{\pi R^{2}} .
$$

After substituting the value of flow rate into equation (29), so the average velocity will be

$$
\bar{V}=\frac{n}{1+3 n}\left[\left(\frac{\rho g}{2 \eta L}(H(t)+L)\right)^{\frac{1}{n}} R^{\frac{1}{n}+1}+\frac{\varepsilon}{2(1+2 n)}\left(\frac{\rho g}{2 \eta L}(H(t)+L)\right)^{\frac{2}{n^{-1}}} R^{\frac{2}{n}+2}\right] .
$$

Mass balance over the entire tank is

$$
\frac{d}{d t}\left[\pi R_{T}^{2} H(t)\right]=-Q(t)
$$

Substituting flow rate from equation (28) into equation (31) and then separating variables on both sides of equation one obtains

$$
\left[\begin{array}{l}
\left(\frac{\rho g}{2 \eta L}\right)^{-\frac{1}{n}} R^{-\frac{1}{n}-3}\left\{(H(t)+L)^{1-\frac{1}{n}}-\left(H_{0}+L\right)^{1-\frac{1}{n}}\right\}-\frac{\varepsilon}{2(1+2 n) R^{2}}\left(\frac{\rho g}{2 \eta L}\right)^{-7} \times \\
\ln \left\{\begin{array}{l}
(H(t)+L)^{1-\frac{1}{n}}+\frac{\varepsilon R^{\frac{1}{n}+1}}{2(1+2 n)}\left(\frac{\rho g}{2 \eta L}\right)^{\frac{1}{n}-1} \\
\left(H_{0}+L\right)^{1-\frac{1}{n}}+\frac{\varepsilon R^{\frac{1}{n}+1}}{2(1+2 n)}\left(\frac{\rho g}{2 \eta L}\right)^{\frac{1}{n}-1}
\end{array}\right\}
\end{array}\right]=\frac{t(1-n)}{R_{T}^{2}(1+3 n)}
$$


and the time required for complete drainage is obtained by taking $H(t)=0$ in $\frac{R_{T}^{2}(1+3 n)}{(1-n)}\left[\begin{array}{l}\left(\frac{\rho g}{2 \eta L}\right)^{-\frac{1}{n}} R^{-\frac{1}{n}-3}\left\{(H(t)+L)^{1-\frac{1}{n}}-\left(H_{0}+L\right)^{1-\frac{1}{n}}\right\}-\frac{\varepsilon}{2(1+2 n) R^{2}}\left(\frac{\rho g}{2 \eta L}\right)^{-7} \times \\ \ln \left\{\begin{array}{l}(H(t)+L)^{1-\frac{1}{n}}+\frac{\varepsilon R^{\frac{1}{n}+1}}{2(1+2 n)}\left(\frac{\rho g}{2 \eta L}\right)^{\frac{1}{n}-1} \\ \left(H_{0}+L\right)^{1-\frac{1}{n}}+\frac{\varepsilon R^{\frac{1}{n}+1}}{2(1+2 n)}\left(\frac{\rho g}{2 \eta L}\right)^{\frac{1}{n}-1}\end{array}\right\}\end{array}\right]=t$

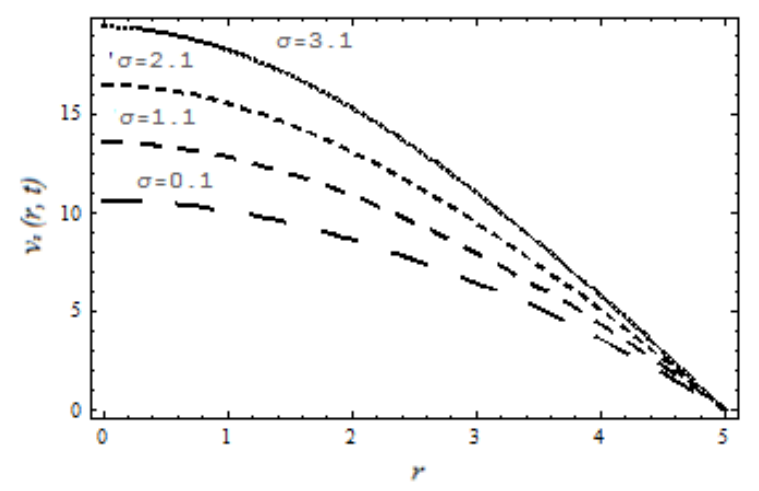

Figure 2: Effect of $\sigma$ on velocity profile,

when $\eta=11.5$ poise, $\rho=0.78 \mathrm{~g} / \mathrm{cm}^{3}, n=1.2$ $R=5 \mathrm{~cm}, L=10 \mathrm{~cm}, B_{0}=1, H(t)=20 \mathrm{~cm}$.

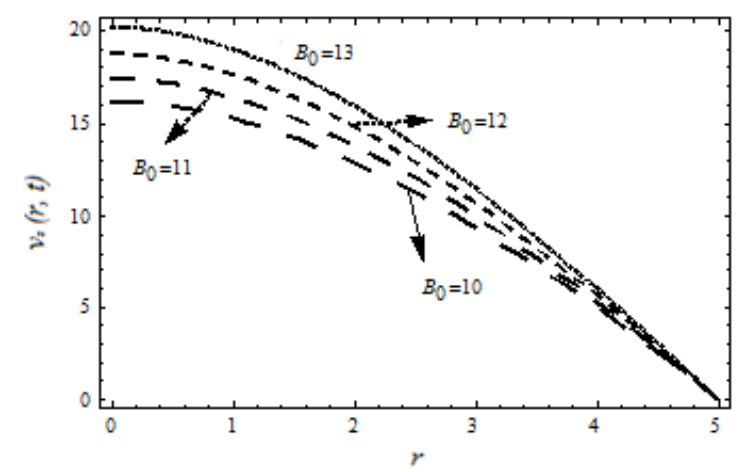

Figure 3: Effect of $B_{0}$ on velocity profile, 
when $\eta=11.5$ poise, $\rho=0.78 \mathrm{~g} / \mathrm{cm}^{3}, n=1.2$, $R=5 \mathrm{~cm}, L=10 \mathrm{~cm}, \sigma=0.02, H(t)=20 \mathrm{~cm}$.

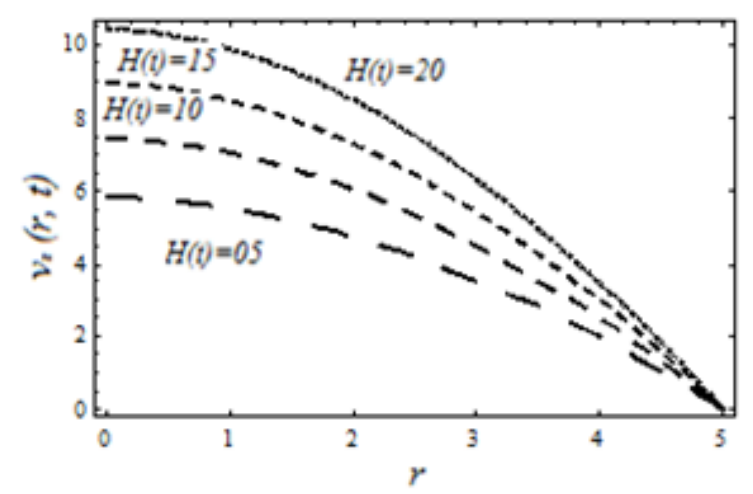

Figure 4: Effect of $H(t)$ on velocity profile, when $\eta=11.5$ poise, $\rho=0.78 \mathrm{~g} / \mathrm{cm}^{3}, n=1.2$ $R=5 \mathrm{~cm}, L=10 \mathrm{~cm}, \sigma=0.1, B_{0}=0.25$.

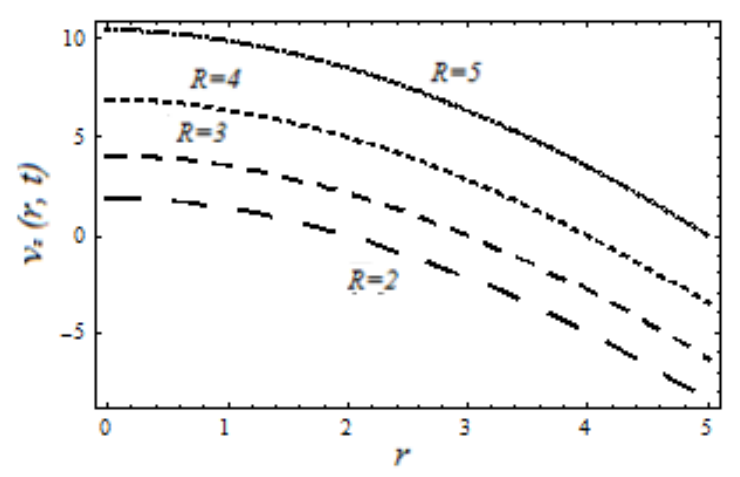

Figure 5: Effect of $R$ on velocity profile, when $\eta=11.5$ poise $\rho=0.78 \mathrm{~g} / \mathrm{cm}^{3}, n=1.2$ $L=10 \mathrm{~cm}, H(t)=20 \mathrm{~cm}, \sigma=0.1, B_{0}=0.25$.

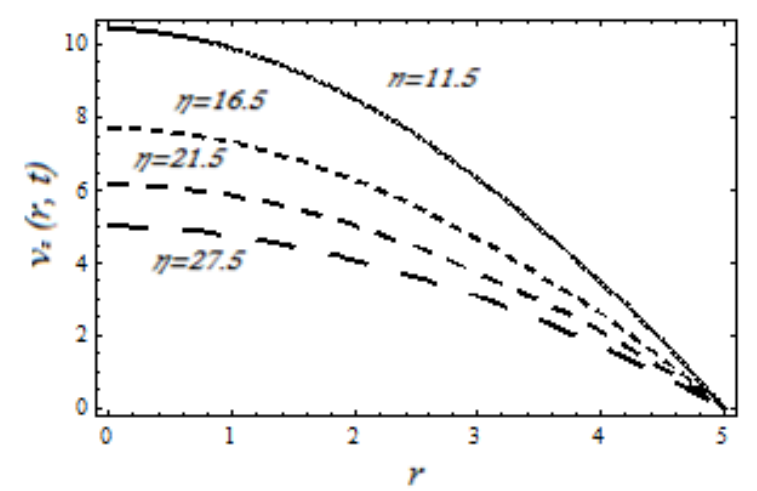

Figure 8: Effect of $\eta$ on velocity profile,

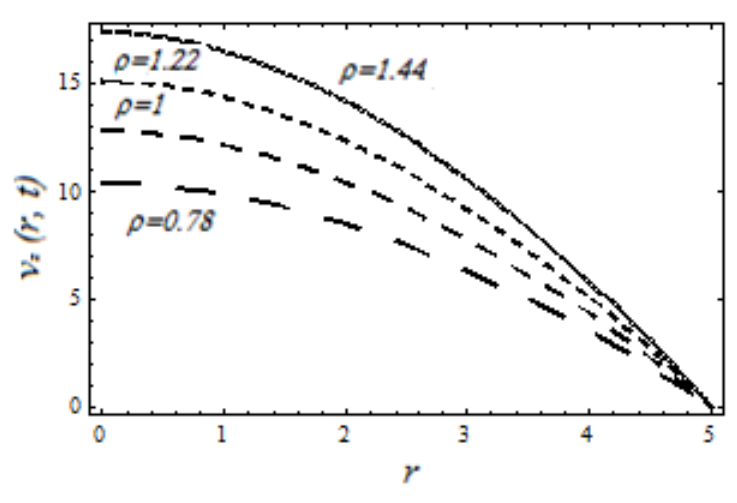

Figure 6: Effect of $\rho$ on velocity profile, when $\eta=11.5$ poise, $R=5 \mathrm{~cm}, n=1.2$, $L=10 \mathrm{~cm}, H(t)=20 \mathrm{~cm}, \sigma=0.1, B_{0}=0.25$.

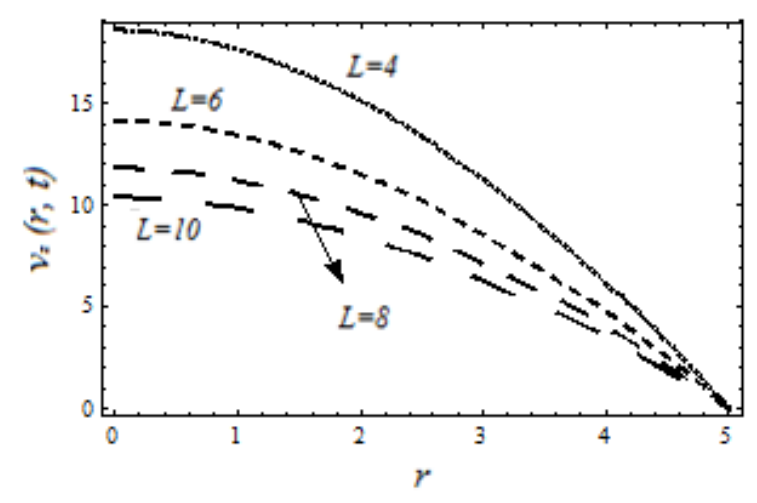

Figure 7: Effect of $L$ on velocity profile, when $\eta=11.5$ poise, $\rho=0.78 \mathrm{~g} / \mathrm{cm}^{3}, n=1.2$ $R=5 \mathrm{~cm}, H(t)=20 \mathrm{~cm}, \sigma=0.1, B_{0}=0.25$.

when $\rho=0.78 \mathrm{~g} / \mathrm{cm}^{3}, R=5 \mathrm{~cm}, L=10 \mathrm{~cm}$, $H(t)=20 \mathrm{~cm}, \sigma=0.1, B_{0}=0.25, n=1.2$. 


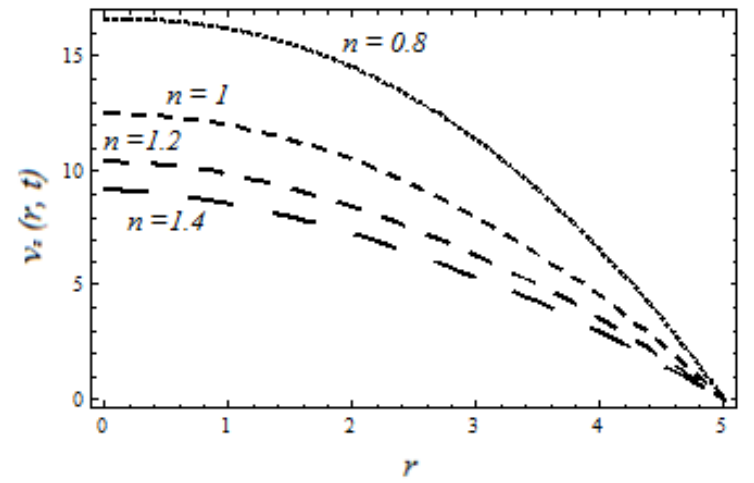

Figure 9: Effect of $n$ on velocity profile, when $\rho=0.78 \mathrm{~g} / \mathrm{cm}^{3}, R=5 \mathrm{~cm}, L=10 \mathrm{~cm}$, $H(t)=20 \mathrm{~cm}, \sigma=0.1, B_{0}=0.25, \eta=11.5$.

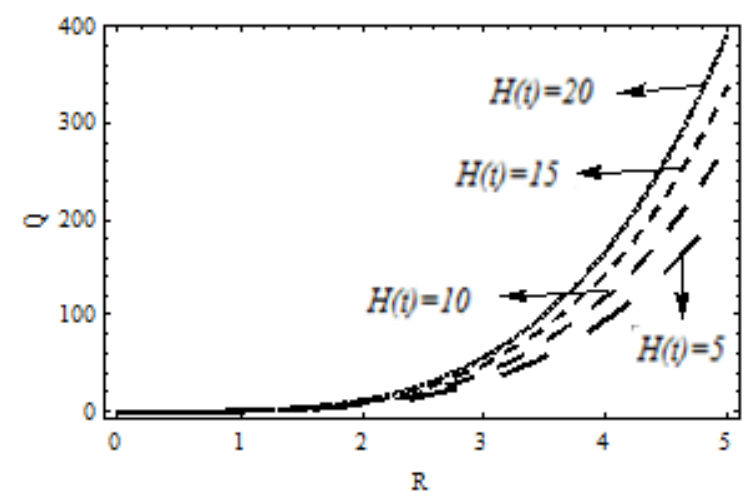

Figure 10: Effect of $H(t)$ on flow rate, when $\eta=11.5$ poise, $\rho=0.78 \mathrm{~g} / \mathrm{cm}^{3}, L=10 \mathrm{~cm}$, $\sigma=0.1, n=1.2, B_{0}=0.25$.

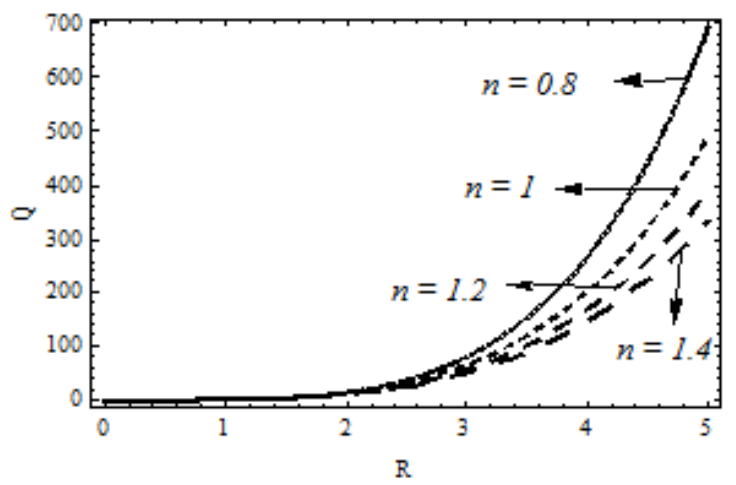

Figure 11: Effect of $n$ on flow rate, when

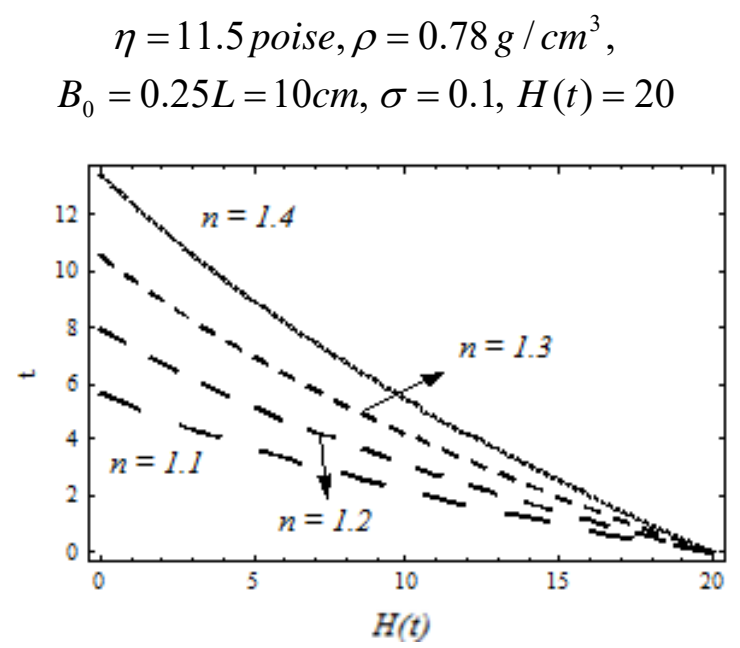

Figure 12: Effect of $n$ on time w. r. to depth, when $\eta=0.6 p o i s ̧ e \rho=1.38 \mathrm{~g} / \mathrm{cm}^{3}, \sigma=0.1$ , $H_{0}=20 \mathrm{~cm}, L=10 \mathrm{~cm}, R=5, B_{0}=0.25, R_{T}=25$

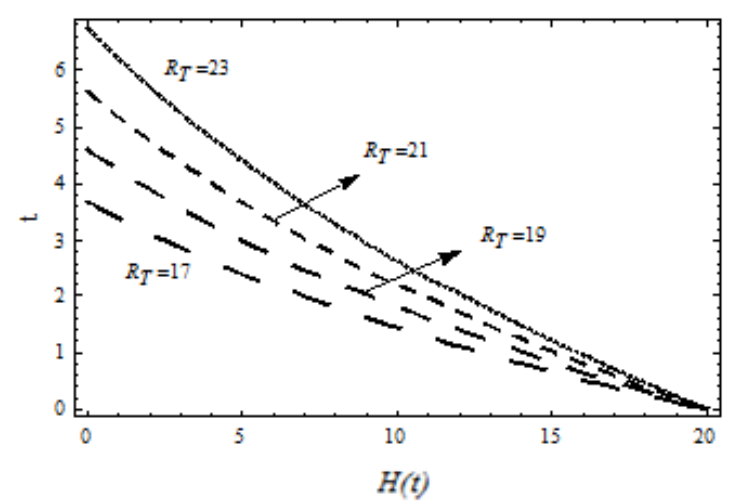

Figure 13: Effect of $R_{T}$ on time w. r. to depth, when $\eta=0.6$ poise, $\rho=1.38 \mathrm{~g} / \mathrm{cm}^{3}$, $\sigma=0.1, H_{0}=20 \mathrm{~cm}, L=10 \mathrm{~cm}, R=5, B_{0}=0.25, n=1.2$ 


\section{Results and discussion}

In the overhead sections we contemplated tank drainage problem utilizing an incompressible Power law MHD fluid, Analytical solution's for the nonlinear differential equation is acquires by using perturbation method. The variation of velocity profile $v_{z}$, flow rate $Q$ and time $t$ required for to complete drainage has been investigated on different parameters. The effects of the electrical conductivity $\sigma$, applied magnetic field $B_{0}$, dynamic viscosity $\eta$, depth $H(t)$, length of pipe $L$, pipe radius $R$, density $\rho$ and for Power law index $n$ on velocity profile are observed through figures (2) - (9) as well as effect of the depth $H(t)$ and Power law index $n$ on flow rate are shown in figures (10) - (11) and effect of the radius of tank $R_{T}$ as well as Power law index $n$ on on time $t$ required to complete drainage is examined in figure (12) - (13). In figures (2) - (9) it is detected that the magnitude of velocity increases as the expansion of electrical conductivity $\sigma$, applied magnetic field $B_{0}$, depth $H(t)$, pipe radius $R$ and density $\rho$ and decreases for the increase of length of pipe $L$, dynamic viscosity $\eta$ and Power law index $n$. From figure (9) we can summarized that as the fluid is becoming thinner the magnitude of velocity increases. In figures (10) - (11) for the increase $H(t)$ we detected that flow rate increases and decrease for increasing $n$. Figures (12) - (13) are plotted for the time $t$ required for to complete drainage with respect to depth, we point out that with increase of radius of $\operatorname{tank} R_{T}$ and power law index $n$ then it will take a time for completely drain from the tank. It is evident from figure (12) that fluid descends more quickly as the value of $n$ decreases.

\section{Conclusions}

We have presented results for unsteady, incompressible, isothermal tank drainage flow for the Power law MHD fluid and obtained exact solutions for "velocity profile, flow rate, average velocity, relation of depth of the tank and time required for complete drainage". Here it is noted that for the perturbation parameter $\varepsilon=0$, solution of the problem reduces to Power law fluid [11] and for substituting $\varepsilon=0$ and $n=1$, we recover the solution concerning to Newtonian withought MHD fluid [28]. A relationship (33), how does the time shift with length is inferred. It is noticed that as the fluid is getting to be thicker, velocity of the fluid decreases and thinner for taking velocity vice versa. 


\section{References}

[1] N. S. Deshpande, M. Barigou, Vibrational flow of non-Newtonian fluids, Chemical Engineering Science, 56, 3845-3853 (2001).

[2] M. Kemiha, X. Frank, S. Poncin, H. Z. Li, Origin of the negative wake behind a bubble rising in non-Newtonian fluids, Chemical Engineering Science, 61, 4041-4047 (2006).

[3] Memon, K. N., S. Islam, A. M. Siddiqui, S. A. Khan, N. A. Zafar and M. Akram, Lift and drainage of Electrically Conducting Power Law Fluid on a Vertical Cylinder, International Journal Applied Mathematics and Information Sciences, Appl. Math. Inf. Sci. 8, No. 1, 45-55 (2014).

[4] A. M. Siddiqui, M. Akram, K. N. Memon, S. Islam and Khalid khan, Withdrawal and drainage of thin film flow on a vertical Cylinder, Scientific Research and Essays, 7, 1992-2248 (2012).

[5] J. N. Kapur, Flows of Power-Law Fluid Past a Flat Plate with uniform suction and between two parallel plates with uniform suction and injection, Journal of the physical society of Japan, 18, 578-581 (1963).

[6] Joe Leonared, S.T. Macklin, Jennifer Ogunyomi, Tank drainage modeling, Oklahoma State University, School of Chemical Engineering, Unit operation laboratory 3/25/09

[7] E. J. Crosby, Experiments in Transport Phenomena, Wiley, New York, (1961).

[8] R. B. Bird, W. E. Stewart, and E. N. Lightfoot, Transport Phenomena, Wiley, New York, (1960).

[9] D. Hanesian, Chemical Engineering Laboratory Manual, NJIT, Newark, (1984).

[10] De Nevers, N., "Fluid Mechanics for Chemical Engineers", 2nd ed., McGrawHill, New York, NY. p.164, (1991).

[11] Bird, R.B., W.E. Stewart and E.N. Lightfoot, "Transport Phenomena", 2nd ed., John Wiley \& Sons, New York, NY, pp. 199-200, 216-218, 228-229, (2002).

[12] Donald D. Joye and Branden C. Barrett, The Tank Drainage Problem Revisited:

Do These Equations Actually Work?The Canadian Journal of Chemical Engineering, Volume 81, October (2003) 
[13] Wilkes, J.O. "Fluid Mechanics for Chemical Engineers", Prentice-Hall PTR, Upper Saddle River, NJ,. p.68, (1999).

[14] David b. Van dongen, Efflux Time from Tanks with Exit Pipes and Fittings, Int. J. Engng Ed. Vol. 15, No. 3, pp. 206 \pm 212 , (1999).

Josue' Njock Libiia, Mechanics of the slow draining of a large tank under gravity, Am. J. Phys. $71 \sim 11$ !, November, (2003).

[15] Lawrence K. Forbes, Unsteady draining flows from a rectangular tank, Physics of Fluids 19, 082104, (2007).

[16] Lawrence K. Forbes, Graeme C. Hocking, Unsteady draining of a fluid from a circular tank, Applied Mathematical Modelling 34 3958-3975, (2010).

[17] Ch. V. Subbarao, Comparison of Efflux Time between Cylindrical and Conical Tanks Through an Exit Pipe International Journal of Applied Science and Engineering. 9, 1: 33-41, (2011).

[18] A. Uma Devi, P.V. Gopal Singh, G.V.S.K. Reddy, S.J. Dharwal and C.H.V. Subbarao, A Review on Efflux Time, Middle-East Journal of Scientific Research 9 (1): $57-63,(2011)$.

[19] G.V.S.K.Reddy, Ch.V.Subbarao, Comparison of efflux times between cylindrical and spherical Tank through an exit pipe, International Journal of Engineering \& Applied Sciences (IJEAS) Vol.3, Issue 261-68, (2011).

[20] A. Uma Devi, D. V. Padma \& CH. V. Subbarao, Effect of Polymer Solutions on Efflux Time for Two Exit Pipe System, Vol. 1 No. 1 September-November, (2011)

[21] Ch.V.Subbarao, P.Srinvasa Rao, G.M.J.Raju and V.S.R.K.Prasad, Slow draining of large spherical tank under gravity, Elixir Chem. Engg. 50,10346-10348, (2012).

[22] Ch. V. Subbaraoa, Madhavi, D. Appala Naidu, and P. King, Use of polymer solutions for drag reduction in gravity driven flow systems, International Journal of Applied Science and Engineering. 11, 2: 159-169, (2013).

[23] Subbarao, Chirravuri Venkata, Drag reduction by surfactant solutions in gravity driven flow systems, Iran. J. Chem. Chem. Eng. Vol. 32, No. 2, (2013) 
[24] Mohyuddin MR, Gotz T. Resonance behavior of viscoelastic fluid in Poiseuille flow in the presence of a transversal magnetic field. International Journal for numerical methods in fluids, 49, p. 837-847(2005).

[25] Rossow VJ. On flow of electrically conducting fluids over a flat plate in the presence of a transverse magnetic field, NASA, Report no. 1358, 489, 1958.

[26] Pop I., Kumari M., Nath G. Conjugate MHD flow past a flat plate, Acta Mech. 106: 215-220 (1994).

[27] Abel S., Veena PH., Rajagopal KR., Prayin VK. Non-Newtonian magnetohydrodynamic flow over a stretching surface with heat and mass transfer, Int. J. Non-Linear Mech. 39:1067-1078(2004).

[28] T. C. Papanastasiou, Applied Fluid Mechanics, P T R Prentice Hall (1994). 